Editorial

\title{
Acknowledgment to Reviewers of CivilEng in 2021
}

\author{
CivilEng Editorial Office
}

MDPI AG, St. Alban-Anlage 66, 4052 Basel, Switzerland

Rigorous peer-reviews are the basis of high-quality academic publishing. Thanks to the great efforts of our reviewers, CivilEng was able to maintain its standards for the high quality of its published papers. Thanks to the contribution of our reviewers, in 2021, the median time to first decision was 24.5 days and the median time to publication was 54 days. The editors would like to extend their gratitude and recognition to the following reviewers for their precious time and dedication, regardless of whether the papers they reviewed were finally published:

Abid Nadeem

Agnieszka Leśniak

Akanshu Sharma

Alessandro Grazzini

Amr M. Morsy

Angelo Aloisio

Annemarie Muntendam-Bos

Axel Hahn

Bartolomeo Coppola

Bartolomeo Megna

Bassam A. Tayeh

Bernardo Luís Filipe Almeida

Camelia Cerbu

César García Lozano

Cesare Oliviero Rossi

Charles Sprouse

Chengping Zhang

Chris G. Karayannis

Cynthia Hou

Davide Forcellini

Dimitrios E. Koulouriotis

Dimitris Theodossopoulos

Eduardo Faleiro

Ehsan Harirchian

Elżbieta Radziszewska-Zielina

Emiliano Pasquini

Eugeniusz Koda

Fabio Favoino

Filippo Masi

Francesco Colangelo

Frans Bijlaard

Gaetano Falcone

George D. Hatzigeorgiou

George Papagiannopoulos
Getu Hailu

Gian Piero Lignola

Giorgio De Alteriis

Giorgio Ficco

Giovanni Muciaccia

Glikeria Kakali

Greg White

Grzegorz Lesiuk

Grzegorz Ludwik Golewski

Hailong Ye

Haridharan Kanthamani

Hassan Karampour

Ibukun Gabriel Awolusi

Indre Grazuleviciute-Vileniske

Isabelle Demers

Jarosław Rybak

Javier J. Cancela

Joacim Lundberg

Jörg Reiff-Stephan

José Ricardo Carneiro

Kai Wei

Kaveh Zamani

Ke Chen

Kleopatra Petroutsatou

Kourosh Mohammadi

Kresimir Nincevic

Krzysztof Zima

Lech Lichołai

Li Hui

Lily Poulikakos

Lucia Figuli

Ludger Lohaus

Magued Iskander

Małgorzata Pająk 
Mamzi Afrasiabi

Marcus Sandberg

Maria Bostenaru Dan

Mark H. Wayne

Marko Vauhkonen

Mateusz Iwański

Michele Betti

Miloš Marjanović

Mislav Stepinac

Mostafa Razzaghmanesh

Muhammad Javed

Muhammad Nasir Amin

Naphol Yoobanpot

Nicola Baldo

Nikolaos Karathanasopoulos

Norena Martin-Dorta

Norimi Mizutani

Nuha Mashaan

Nuno Monteiro Azevedo

Panagiotis Spyridis

Paulo Cachim

Pavel Krivenko

Pedro Jose Martin Moreta
Pietro Crespi

Piotr Borowski

Piotr Jaskuła

Piotr Mioduszewski

Piyaruwan Perera

Riccardo De Benedictis

Ricky Chan

Roberto Nascimbene

Rolf Eligehausen

Roman Wan-Wendner

S. Amir Reza Beyabanaki

Sadegh Ghavami Jamal

Saleh Seyedzadeh

Sánchez Antonio Bernardo

Serdar Durdyev

Seyed Amid Tahami

Seyed Ghaffar

Shriful Islam

Silvino Dias Capitão

Tengfei Fu

Yingsong Gu

Zeman Oliver 